\title{
Le mal-être de l'animal malade et sa gestion en élevage
}

\author{
N. BAREILLE \\ Ecole Nationale Vétérinaire, Unité Zootechnie Economie, BP 40706, F-44307 Nantes, France \\ Courriel : bareille@vet-nantes.fr
}

La maladie, altération de la santé (état de l'individu dont l'organisme fonctionne normalement), est considérée comme une atteinte majeure au bien-être des animaux (Fitzpatrick et al 2006) dont il y est fait référence dans les définitions courantes du bien-être pour les animaux d'élevage. De ce fait, la mesure des troubles de santé fait généralement partie des études de comparaison de conduites d'élevage ou de systèmes de logement des animaux d'élevage. Le trouble de santé est une des conséquences de l'exposition de l'animal à des facteurs de risque (voir articles dans ce numéro). Cependant, peu d'études scientifiques ont cherché à objectiver ce qui semble être une évidence : l'animal malade n'est pas en situation de bienêtre. Cela tient vraisemblablement au fait que la science vétérinaire clinique date d'une époque où le bien-être animal était encore négligé. Cet article a pour objectif d'amener des éléments de réflexion sur les conséquences de la maladie sur le bien-être des animaux et d'envisager les principales voies d'amélioration en élevage.

\section{$1 /$ Répercussions physiolo- giques et conséquences sur le bien-être}

Les troubles de santé des animaux découlent de l'altération des fonctions physiologiques, avec des variations des fonctions modifiées selon leur étiologie. Le modèle le plus simple et aussi le plus fréquent est celui d'une maladie infectieuse à expression clinique (ex : mammite clinique, infections respiratoires aiguës...) où l'agression par un agent pathogène se traduit par une réaction inflammatoire locale ou généralisée chez l'hôte. Par définition, l'inflammation est à l'origine de douleur, mais même en l'absence de réaction inflammatoire, une douleur peut être ressentie, notamment lors de certaines maladies métaboliques telles que les myopathies nutritionnelles.
Lors de la réaction inflammatoire, la libération locale de médiateurs de l'inflammation stimule les nocicepteurs et le stimulus douloureux généré est véhiculé par les fibres nerveuses sensorielles afférentes via la moelle épinière jusqu'au cerveau. L'intensité de la douleur dépend de la sévérité de l'atteinte des tissus et des voies nerveuses (Fitzpatrick et al 2006).

Plusieurs types de méthodes expérimentales ont été développés pour objectiver l'intensité de l'inflammation ou de la douleur engendrée par ces modifications physiologiques. Elles sont en théorie intéressantes chez les animaux d'élevage qui sont des «espèces proies» et qui de ce fait expriment moins leur douleur par des comportements modifiés que les «espèces prédateurs» (Weary et al 2006).

Ainsi, il est possible de mesurer 1'activité nerveuse des nerfs afférents. Il a été montré, lors de castration des agneaux par pose d'élastique autour du scrotum, que l'activité nerveuse des nerfs afférents était augmentée traduisant la stimulation des nocicepteurs locaux (Cottrell et Molony 1995). L'activation du système nerveux sympathique est une constante lors de douleur mais elle est aussi rencontrée lors de stress, ou plus couramment, lors d'activité physique intense. La mesure de la fréquence cardiaque et des concentrations plasmatiques ou salivaires de corticoïdes ont donc peu d'intérêt pour objectiver la douleur chez l'animal en élevage (Molony et Kent 1997).

La mesure des protéines de phase aiguë de l'inflammation offre de nouvelles perspectives d'application en élevage. Il s'agit de protéines sériques dont la concentration est modifiée lors d'inflammation, par exemple l'haptoglobuline, le fibrinogène la céruloplasmine et le sérum amyloïde A (Murata et al 2004). Selon les protéines, les maladies et les espèces étudiées, ces indicateurs peuvent être plus précoces pour la détection des maladies que l'apparition des signes cliniques, et utilisables pour évaluer l'intensité de l'inflammation chez les animaux atteints de maladie sub-clinique (Petersen et al 2004). Cependant, leur dosage reste problématique et cet outil reste, pour le moment, confiné à des explorations expérimentales.

Enfin il existe des méthodes basées sur la quantification des modifications comportementales de l'animal malade (cf. infra). Elles ont permis de montrer que la douleur ne se limite pas strictement à la zone touchée par un processus inflammatoire. En effet, par application d'un stimulus mécanique d'intensité croissante, il a été montré que des vaches laitières ayant une mammite clinique dans un quartier de la mamelle réagissaient plus rapidement s'il était appliqué sur le membre postérieur du côté du quartier atteint que sur celui de l'autre côté. Cette réponse exacerbée en cas de douleur d'origine inflammatoire définit l'hyperalgésie. De plus, l'hyperalgésie se manifeste sur une durée plus longue que les signes cliniques repérables sur la mamelle : jusqu'à 20 jours d'hyperalgésie pour une mammite clinique guérie en quelques jours (Fitzpatrick et al 1999). Une hyperalgésie durable a aussi été démontrée en cas de boiterie chez les bovins (Whay et al 1997) et les ovins (Ley et al 1995).

Outre la douleur, la réaction inflammatoire induit une augmentation de la température corporelle chez l'animal malade. Ceci a pour conséquence d'augmenter les déperditions de chaleur du corps de l'animal qui devra à terme lutter contre le froid. Un environnement d'élevage acceptable pour un animal en bonne santé peut ainsi devenir inconfortable pour un animal malade. 


\section{2 / Répercussions compor- tementales et conséquences sur le bien-être}

L'animal peut présenter diverses modifications comportementales associées à la maladie. Elles permettent au propriétaire de l'animal malade de le repérer et elles servent de base à l'examen clinique du vétérinaire. L'ensemble des signes cliniques présentés par un animal permet de définir un tableau clinique qui est plus ou moins typique d'une affection donnée. Par exemple, un animal ayant une insuffisance respiratoire aiguë restera immobile, les membres antérieurs écartés, l'encolure étendue et la bouche ouverte (syndrome de détresse respiratoire, photo 1) afin d'éviter la compression de la trachée et de la cage thoracique. Un animal ayant une péritonite restera debout, ventre levretté, dos voûté, pattes regroupées sous lui qui piétinent et exprime des plaintes (photo 2). C'est sur la description d'un tableau clinique que repose l'éthologie clinique vétérinaire (Fraser et Broom 1997).

La majeure partie des modifications comportementales visibles sur un animal malade ou blessé se développe en réponse à la douleur. Il peut s'agir (Molony et Kent 1997) de

- mouvements de retrait, le plus souvent réflexes, qui permettent de proté-

Photo 1. Veau présentant un syndrome de détresse respiratoire.

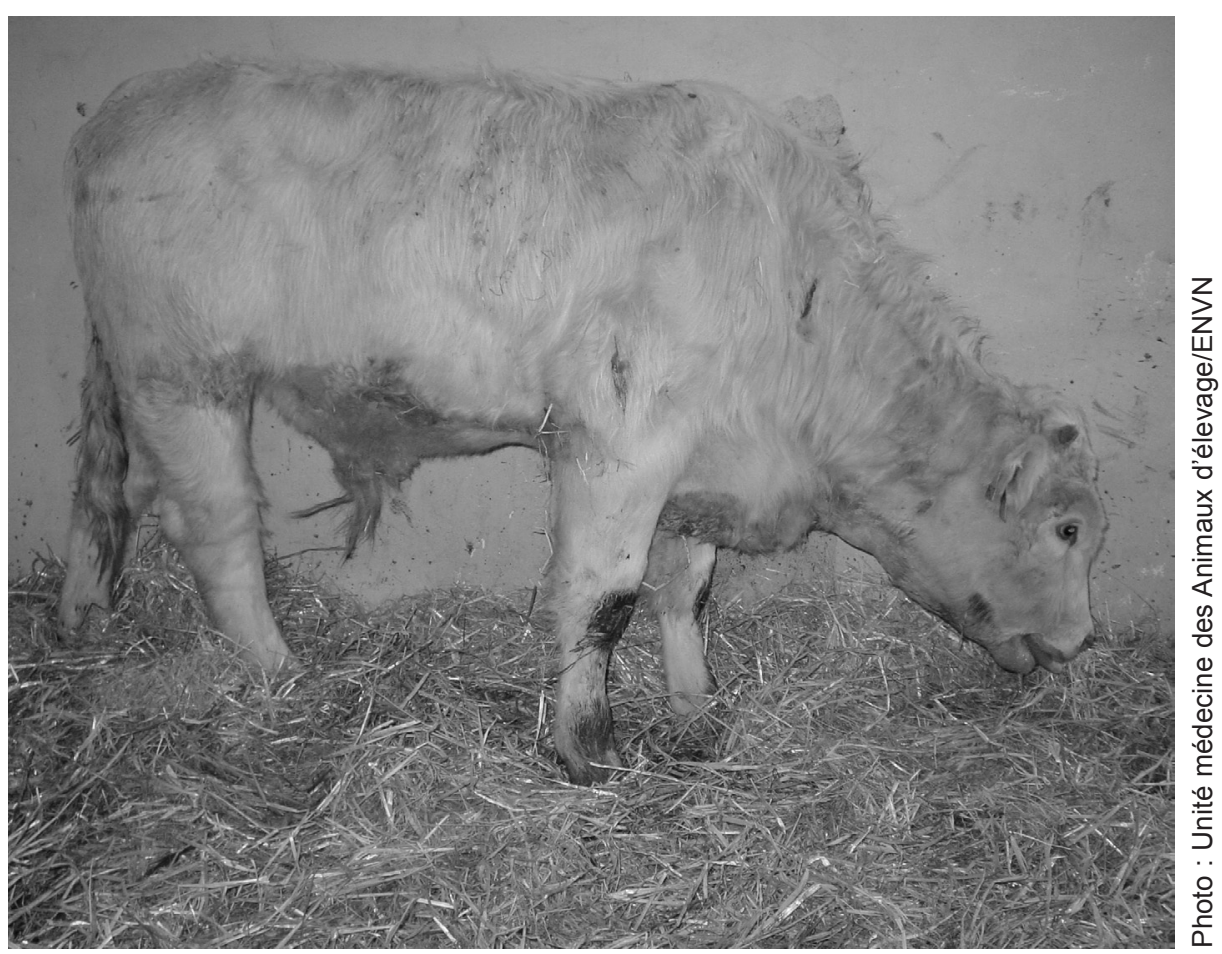

Photo 2. Bovin présentant des signes de péritonite. ger la partie du corps lésée (par exemple les tentatives d'échappement lors de la palpation d'une zone douloureuse),

- comportements et postures modifiés afin d'atténuer la douleur présente et de favoriser indirectement la cicatrisation de la lésion (par exemple le comportement de boiterie),

- comportements de communication envers les congénères (vocalisations,

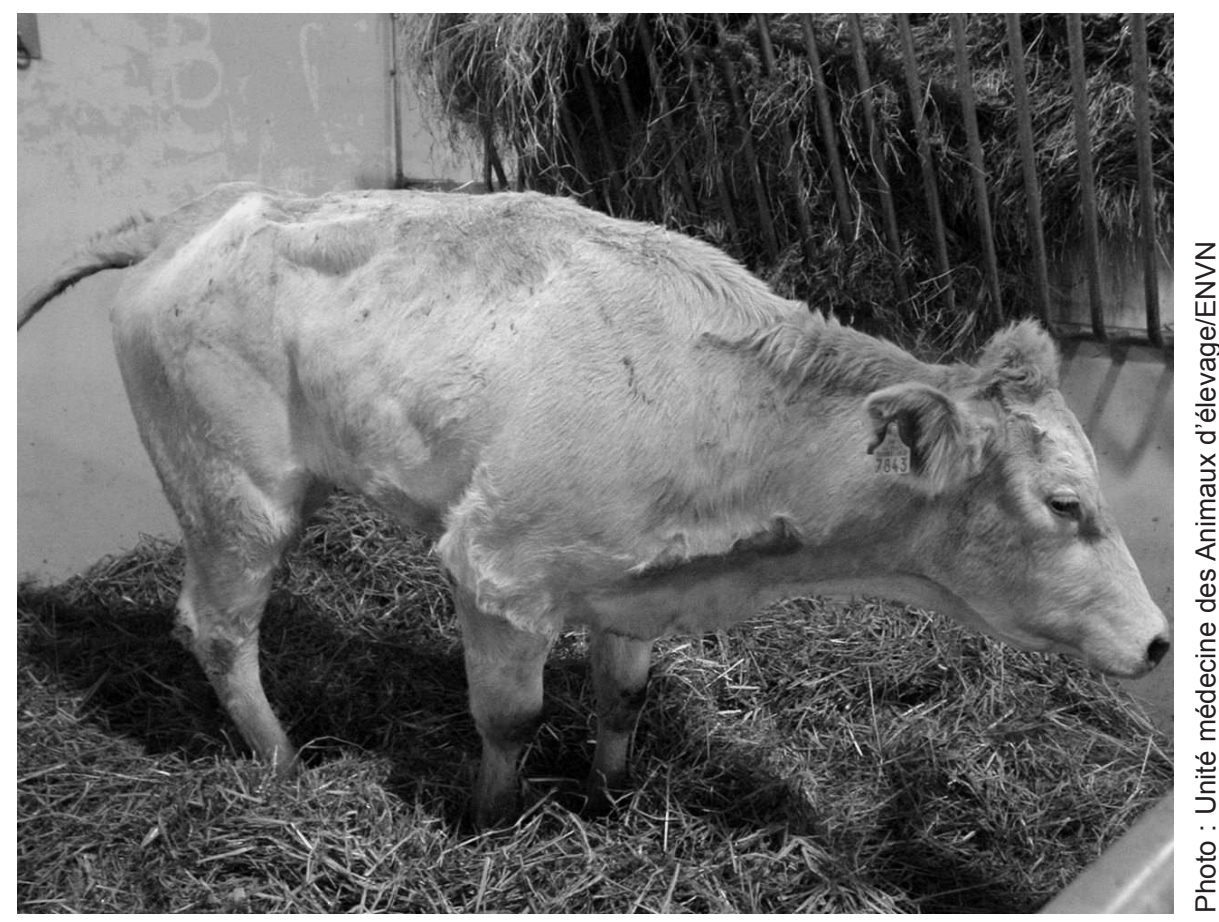

postures et odeurs) afin de solliciter leur aide ou de les éloigner.

Les autres modifications comportementales repérables sont des signes nerveux d'excitation, d'incoordination ou de dépression sans composante douloureuse (Fraser et Broom 1997).

Sur la base de la description du tableau clinique de certaines maladies, Fraser et Broom (1997) montrent que la douleur générée est très variable en intensité et en durée, mais aussi qu'il est difficile de l'évaluer par une observation $\mathrm{du}$ comportement quand la lésion gêne peu les mouvements de l'animal (mammites cliniques par exemple). L'évaluation de l'intensité des signes cliniques comportementaux est en effet subjective. Malgré tout, certaines mesures subjectives sont performantes, notamment celles basées sur des scores cliniques dont la répétabilité intra-observateur et inter-observateurs a été évaluée. Ceci a surtout été fait pour le comportement de boiteries (Welsh et al 1993, Sprecher et al 1997). Les scores cliniques sont des outils faciles à utiliser en élevage et les éleveurs peuvent se les approprier sans difficultés.

En plus de manifester des signes de douleur, le bien-être de l'animal malade est compromis par la difficulté à réaliser ses comportements normaux. Ainsi, il se déplacera moins et aura moins accès à la nourriture et à l'abreuvement, ce qui pourra aggraver l'intensité 
de sa maladie. Du fait de sa faiblesse momentanée, sa position hiérarchique dans le troupeau peut être modifiée par agression de congénères auparavant dominés.

La conséquence de toutes les atteintes physiques au bien-être soulignées précédemment est que l'animal a une production diminuée. La mesure des modifications de performances zootechniques (ingestion, croissance, reproduction, production laitière et longévité) est un outil classique d'évaluation du bien-être ; il peut également être utilisé pour juger de la contribution relative des différentes maladies au mal-être de l'animal. Les études de type épidémiologique sont particulièrement utiles pour quantifier l'effet des troubles de santé sur les performances : à partir des enregistrements de l'occurrence des troubles de santé et des performances animales (Contrôle Laitier, Centre d'Insémination Artificielle) réalisés dans de grands échantillons, il est possible d'estimer l'effet moyen des maladies, par modèles de régression multivariés, et la variabilité de cet effet. Le tableau 1 synthétise les connaissances obtenues chez la vache laitière pour les maladies les plus fréquentes. Elles affectent principalement l'ingestion (maladies métaboliques), la production laitière (déplacement de caillette, mammite clinique...), la reproduction (dystocie, non-délivrance, métrite) ou la longévité (mammite clinique, dystocie, déplacement de caillette). Au global, trois maladies ont un impact zootechnique marqué : déplacement de caillet- te, mammite clinique et boiterie. Une étude des effets zootechniques des troubles de santé a pu être réalisée dans une ferme expérimentale INRA disposant d'enregistrements journaliers d'ingestion et de production de vaches laitières (Bareille et al 2003). Elle a montré que les effets sont visibles dès 4 jours avant le diagnostic clinique et le traitement pour la plupart des maladies. La baisse de production laitière est contemporaine à - et vraisemblablement médiée par - la baisse d'ingestion avec un ratio d'environ $2 \mathrm{~kg}$ de lait perdus pour $1 \mathrm{~kg}$ de matière sèche d'aliment ingéré en moins (équivalence énergétique).

Cette démarche zootechnique permet de juger de la contribution relative des différentes maladies au mal-être de l'animal. Elle peut être complétée par l'approche clinique qui permet d'identifier les maladies les plus douloureuses et durables. Afin de dégager des priorités de lutte contre les maladies les plus pénalisantes pour une production donnée, il faut également intégrer la fréquence de la maladie. Malheureusement, une telle démarche ne peut pas être réalisée actuellement faute de données sur d'autres types d'animaux de production.

\section{3 / Importance du ressenti de l'animal}

L'altération du bien-être physique de l'animal s'accompagne vraisemblablement d'une altération de son bien-être mental : il peut avoir des réactions de peur ou d'anxiété compte tenu de son incapacité à fuir ou à interagir normalement avec ses congénères ; la maladie devient alors une situation stressante pour l'animal. Tout ceci contribue à ce que l'animal malade ressente des difficultés à s'adapter à son environnement (Appleby et Hughes 1997, Fraser et Broom 1997).

La composante psychologique, émotionnelle de la douleur ressentie est parfois niée chez l'animal malade ; les réactions de l'animal face à la douleur seraient purement réflexes... La plupart des arguments en faveur de la composante émotionnelle de la douleur se basent sur l'analogie des structures et voies nerveuses impliquées chez l'homme et l'animal (Bateson 2004). Une expérience faite chez le poulet apporte un argument incontestable (Gentle 2001). Elle consiste à rediriger l'attention de l'animal souffrant d'une affection douloureuse vers une activité a priori plaisante : les auteurs observent des poulets ayant subi une injection de cristaux d'urate de sodium dans l'articulation tibio-métatarsienne qui induit une douleur modérée de quelques heures. Les poulets laissés dans leur cage habituelle sur grillage ne posaient pas leur patte à terre et se déplaçaient avec des signes de boiterie marqués. En revanche, ceux déplacés dans une nouvelle cage avec des copeaux de bois et un nouveau congénère, présentaient des signes de boiteries considérablement atténués.

Tableau 1. Fréquence et effets des principales maladies sur les fonctions de production chez la vache laitière : échelle de 0 (sans effet, peu fréquent) à +++ (effet important, très fréquent).

\begin{tabular}{|c|c|c|c|c|c|}
\hline & Ingestion & \begin{tabular}{|c|}
$\begin{array}{c}\text { Production } \\
\text { laitière }\end{array}$ \\
\end{tabular} & Reproduction $^{1}$ & Longévité $^{2}$ & Fréquence \\
\hline & $\begin{array}{c}\text { Bareille } \\
\text { et a/ } 2003 \\
\end{array}$ & $\begin{array}{l}\text { Fourichon } \\
\text { et al } 1999\end{array}$ & $\begin{array}{l}\text { Fourichon } \\
\text { et al } 2000\end{array}$ & $\begin{array}{l}\text { Beaudeau } \\
\text { et al } 2000 \\
\end{array}$ & $\begin{array}{l}\text { Fourichon } \\
\text { et al } 2001 \\
\end{array}$ \\
\hline Mammite clinique & + & ++ & 0 & ++ & $40-50^{3}$ \\
\hline Boiterie & ++ & ++ & ++ & $0 /+$ & $10-15^{3}$ \\
\hline Dystocie & + & 0 & ++ & ++ & $5-10^{4}$ \\
\hline Non délivrance & 0 & 0 & ++ & 0 & $5-10^{4}$ \\
\hline Métrite & $0 /+$ & $0 /+$ & +++ & + & $5-10^{4}$ \\
\hline Fièvre de lait & ++ & + & + & + & $5-10^{4}$ \\
\hline Cétose clinique & +++ & ++ & + & + & $1-4^{4}$ \\
\hline $\begin{array}{l}\text { Déplacement de } \\
\text { caillette }\end{array}$ & ++ & +++ & 0 & +++ & $0-2^{4}$ \\
\hline
\end{tabular}

\footnotetext{
1 Effet évalué sur la réussite à la première insémination ou sur l'intervalle vêlage - insémination fécondante.

2 Effet évalué sur la durée de vie productive (intervalle premier vêlage - réforme)

3 Exprimé en cas pour 100 vaches et par an.

4 Exprimé en pourcentage des vêlages.
} 
Duncan et Petherick (1991) nuancent tout de même la différence de perception que l'animal et que l'homme peuvent avoir de leur maladie. Chez l'homme, l'état de bien-être physique et mental sont partiellement dissociés : l'homme malade peut se sentir bien, vu qu'il a, entre autres, la capacité de se représenter la fin probable de sa maladie. L'animal a-t-il les capacités cognitives à entrevoir sa guérison ? En corollaire, l'animal a l'avantage de ne pas avoir, en l'absence de signes cliniques, conscience de la maladie, contrairement à l'homme.

\section{4 / Voies d'amélioration de la santé et du bien-être de l'animal malade en élevage}

La première voie d'amélioration possible est bien entendu de prévenir l'apparition des maladies par une maîtrise de la conduite d'élevage et le développement de systèmes de logement adaptés. Les facteurs de risque sont connus pour la plupart des maladies des animaux d'élevage et cela a permis d'élaborer des plans d'intervention dans les élevages présentant des problèmes sanitaires et de concevoir des logements adéquats. Notons que les nouveaux systèmes de logement collectifs pour veaux promus pour améliorer le bien-être des animaux se sont révélés dans les premiers temps de leur utilisa- tion présenter un risque accru de maladies, notamment de diarrhée, par rapport à des systèmes en cases individuelles (Perez et al 1990, Olsson et al 1993). Les éleveurs ont dû apprendre à gérer la santé de leurs animaux dans des conditions difficiles notamment pour le repérage de l'animal malade et pour la réalisation des soins.

C'est en effet le rôle de l'éleveur que de détecter ses animaux malades et de les prendre en charge rapidement et efficacement afin de raccourcir la durée de la maladie, d'alléger leur souffrance et d'améliorer leur confort avec des mesure hygiéniques simples, telles que l'isolement de l'animal malade (photo 3). $\mathrm{Vu}$ l'augmentation de la taille des cheptels, les éleveurs sont demandeurs d'outils de détection précoce des animaux malades. Des outils basés sur la mesure en continu de la fréquence cardiaque et la température corporelle des animaux sont actuellement en cours d'élaboration. Des actions de sensibilisation et de formation des éleveurs sur ce sujet se mettent en place actuellement, comme par exemple l'action de formation «éleveur infirmier de son élevage» proposée aux éleveurs en production bovine conjointement par la Fédération Nationale des Groupements de Défense Sanitaire, le Syndicat National des Vétérinaires d'Exercice Libéral et par la Société Nationale des Groupements Techniques Vétérinaires. Cependant, ces actions ne font pas de l'amélioration du bien-être leur objectif

Photo 3. Bovin malade maintenu dans son lot d'origine et subissant les dérangements de ses congénères.

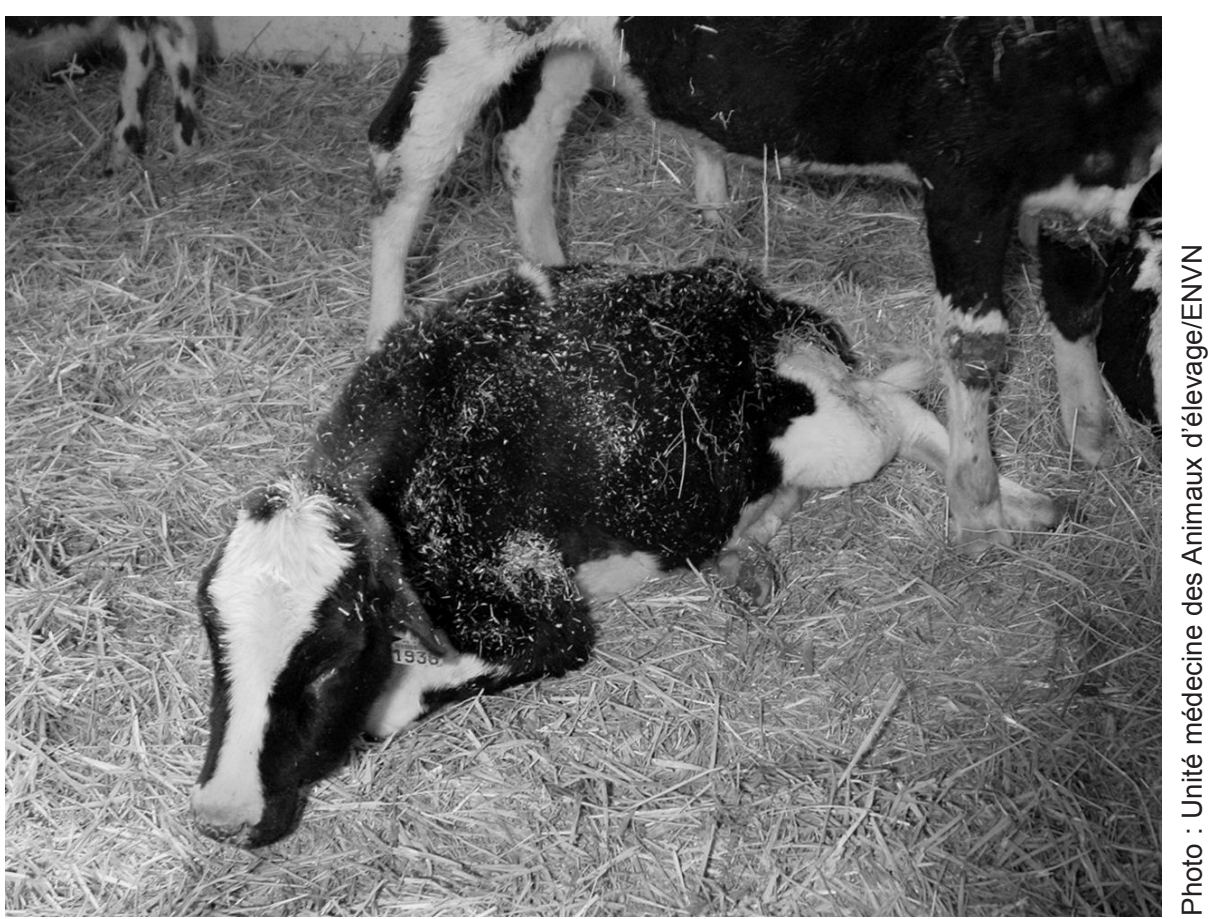

principal, qui est plus de renforcer la sécurité sanitaire des produits d'origine animale.

Le sujet de la décision d'euthanasier les animaux en état de dépérissement avancé est peu évoqué ; l'éleveur doit prendre sa décision sans réellement avoir de consignes claires sur les critères et le moment où cette décision doit être prise. En production porcine aux Etats-Unis, le Swine Welfare Assurance Program recommande un pourcentage minimum, parmi les animaux morts, de $20 \%$ d'animaux euthanasiés et ce de façon à inciter les éleveurs à abréger les souffrances inutiles des animaux en fin de vie.

Des développements plus importants pourraient également être faits sur l'utilisation indispensable des analgésiques, tels que les anti-inflammatoires non stéroïdiens lors de maladies à composante inflammatoire compte tenu de leur efficacité démontrée pour lutter contre la douleur et pour récupérer plus rapidement la capacité de production des animaux (Benson 2004). La sensibilisation et la formation des vétérinaires sur ce sujet est nécessaire : bien qu'ils se disent dans leur grande majorité concernés par la gestion de la douleur chez l'animal, l'utilisation d'analgésiques en suivi post-opératoire chez les carnivores n'est pas une constante (84\% suite à de la chirurgie orthopédique et $17 \%$ suite à une castration) (Hugonnard et al 2004).

Une voie prometteuse d'amélioration du bien-être et de la santé des animaux en ferme est le développement de démarches d'assurance qualité en élevage. En France, elles sont plus centrées sur la qualité que le bien-être, à l'opposé de ce qui existe dans certains pays : le RSPCA's Freedom Food au Royaume-Uni (Main et al 2003), l'Animal Needs Index en Autriche (Bartussek 1999), ou le Human Farm Animal Care aux Etats-Unis (Stull et al 2005). Leur cahier des charges donne des obligations de moyens, c'est-à-dire qu'il décrit les conditions dans lesquelles doivent être élevés les animaux et en particulier les procédures mises en œuvre pour soigner les animaux malades, pour les euthanasier... Ceci ne permet cependant pas de garantir qu'un bon niveau de bien-être est atteint pour les animaux, si l'évaluation se base sur des mesures directes sur l'animal (blessures, état du plumage ou du pelage, boiteries, troubles de santé, état de propreté, état corporel, comportement, réactivité à l'homme). Ainsi, pour un même système 
d'élevage et de logement (moyens identiques), il existe une forte dispersion des mesures faites sur les animaux ce qui indique une implication variable des éleveurs dans le soin apporté aux animaux (Main et al 2003, Mollenhorst et al 2005) et laisse entrevoir des marges de progrès importantes. Notons cependant que les critères de santé sont difficiles à mesurer lors d'une visite ponctuelle en élevage. En effet, lorsque les animaux sont traités de façon efficace, la maladie ne dure que quelques jours. Ainsi, même lorsque l'incidence de la maladie est élevée dans un troupeau, la probabilité d'observer au moins un animal malade est faible et il convient alors de se baser sur les enregistrements de l'éleveur. L'approche par enquête transversale en élevage est tout de même intéressante pour les maladies potentiellement durables et peu prises en charge par les éleveurs telles que les affections podales (Main et al 2003). Dans ce cas, la prévalence de la maladie, c'est à dire le pourcentage d'animaux malades dans un troupeau, peut être un indicateur discriminant des élevages (Toczé 2006).

\section{Conclusion}

La santé est une composante importante du bien-être des animaux : l'animal malade ressent de la douleur, l'exprime par des modifications comportementales manifestes et voit ses performances zootechniques diminuer. Il apparaît nécessaire d'intensifier la sensibilisation et la formation des éleveurs aux soins à délivrer aux animaux malades, et celle des vétérinaires à la prise en compte de la douleur dans les pratiques thérapeutiques.

\section{Références}

Appleby M.C., Hughes B.O., 1997. Animal Welfare. Oxon: Editions CAB international, UK, $336 \mathrm{p}$

Bareille N., Beaudeau F., Billon S., Robert A., Faverdin P., 2003. Effects of health disorders on feed intake and milk production in dairy cows. Livest. Prod. Sci., 83, 53-62.

Bartussek H., 1999. A review of the animal needs index (ANI) for the assessment of animals' well-being in the housing systems for Austrian proprietary products and legislation. Livest. Prod. Sci., 61, 179-192.

Bateson P., 2004. Do animals suffer like us? The assessment of animal welfare. Vet. J. 168, 110-111.

Beaudeau F., Seegers H., Ducrocq V., Fourichon C., Bareille N., 2000. Effect of health disorders on culling in dairy cows: a review and a critical discussion. Ann. Zootech., 49, 293-311.

Benson G.J., 2004. Pain in farm animals: nature, recognition, and management. In: The well-being of farm animals: challenges and solutions, Benson G.J., Rollins B.E. (Eds), Blackwell, Oxford, 61-84.

Cottrell D.F., Molony V., 1995. Afferent activity in the superior spermatic nerve of lambs: the effects of of application of rubber castration rings. Vet. Res. Commun., 19, 503-515.

Duncan I.J.H, Petherick J.C., 1991. The implications of cognitive processes for animal welfare. J. Anim. Sci., 69, 5071-5082.

Fitzpatrick J., Scott M., Nolan A., 2006 Assessment of pain and welfare in sheep. Small Rumin. Res., 62, 56-61.

Fitzpatrick J.L., Young F.J., Eckersall P.D., Logue D.N., Knight C.H., Nolan A., 1999. Mastitis, a painful problem. Cattle Pract., 7, 225226.

Fourichon C., Seegers H., Bareille N., Beaudeau F., 1999. Effects of disease on milk production in the dairy cow: a review. Prev. Vet. Med., 41, 1-35.
Fourichon C., Seegers H., Malher X., 2000. Effect of disease on reproduction in the dairy cow: a meta-analysis. Theriogenology, 53, 17291759

Fourichon C., Beaudeau F., Bareille N., Seegers H., 2001. Incidence of health disorders in dairy farming systems in western France. Livest. Prod. Sci., 68, 157-170.

Fraser A.F., Broom D.M., 1997. Farm animal behaviour and welfare. $3^{\text {rd }}$ Edition, Oxon: Editions CAB international, UK, 448p.

Gentle M.J., 2001. Attentional shifts alter pain perception in the chicken. Anim. Welf., 10, S187-S194.

Hugonnard M., Leblond A., Keroack S., Cadoré J., Troney E., 2004. Attitudes and concerns of French veterinarians towards pain and analgesia in dogs and cats. Vet. Anaesth. Analg., $31,154-163$

Ley S.J., Livingstone A., Waterman A.E., 1995. The effect of chronic clinical pain on mechanical and thermal thresholds in sheep. Vet. Rec., 137, 85-87.

Main D.C.J., Whay H.R., Green L.E., Webster A.J.F., 2003. Effect of the RSPCA freedom food scheme on the welfare of dairy cattle. Vet. Rec. $153,227-231$

Mollenhorst H., Rodenburg T.B., Bokkers E.A.M., Koene P., de Boer I.J.M., 2005. On farm assessment of laying hen welfare: a comparison of one environment-based and two animal-based methods. Appl. Anim. Behav. Sci., 90, 277-291.

Molony V., Kent J.E., 1997. Assessment of acute pain in farm animals using behavioral and physiological measurements. J. Anim. Sci., 75, 266-272.

Murata H., Shimada N., Yoshioka M., 2004. Current research on acute phase proteins in veterinary diagnosis: an overview. Vet. J., 168, 2840.

Olsson S.O, Viring S, Emanuelsson U., Jacobsson S.O., 1993. Calf diseases and morta- lity in Swedish Dairy herds. Acta Vet. Scand., 34(3), 263-269.

Perez E., Noordhuizen J.P.T.M., Van Wuijkhuise L.A., Stassen E.N., 1990. Management factors related to calf morbidity and mortality rates. Livest. Prod. Sci., 25(1-2), 79-93.

Petersen H.H., Nielsen J.P., Heegaard P.M.H., 2004. Application of acute phase proteins measurements in veterinary clinical chemistry. Vet. Res., 35, 163-187.

Sprecher D.J., Hostetler D.E., Kaneene J.B., 1997. A lameness scoring system that use posture and gait to predict dairy cattle reproductive performance. Theriogenology, 47, 1178-1187.

Stull C.L., Reed B.A., Berry S.L., 2005. A comparison of three animal welfare assessment programs on California dairies. J. Dairy Sci., 88, 1595-1600.

Toczé C., 2006. Bien-être des vaches laitières : fréquence de boiteries dans différents systèmes de logement et facteurs de risque impliqués. Mémoire de dominante «Médecine des Animaux d'Elevage et qualité», Ecole Nationale Vétérinaire de Nantes, 84p.

Weary D.M., Niel L., Flower F.C., Fraser D., 2006. Identifying and preventing pain in animals. Appl. Anim. Behav. Sci., sous presse.

Welsh E.M., Gettinby G., Nolan A.M., 1993. Comparison of a visual analogue scale and a numerical rating scale for assessment of lameness, using sheep as a model. Am. J. Vet. Res., $54,976-983$.

Whay H.R., Waterman A.E., Webster A.J.F. 1997. Associations between locomotion, claw lesions and nociceptive threshold in dairy heifers during the peri-partum period. Vet. J., 154, 155161. 


\title{
Résumé
}

L'objectif de cet article est d'amener des éléments de réflexion sur les conséquences de la maladie sur le bien-être des animaux et d'envisager les principales voies d'amélioration en élevage. Le bien-être physique de l'animal malade est compromis : il a des modifications physiologiques comparables à celles liées au stress, il doit adopter des postures et des mouvements anormaux afin de limiter la douleur, il a des difficultés à réaliser ses comportements normaux ce qui se traduit in fine par des baisses de performances. Son bien-être mental est lui aussi affecté. Une sensibilisation des intervenants en élevage au mal-être de l'animal malade doit être entreprise. L'éleveur doit détecter et prendre en charge rapidement ses animaux malades. Les vétérinaires doivent améliorer leurs procédures thérapeutiques en ayant recours dès que nécessaire aux analgésiques.

\begin{abstract}
Suffering of the sick animal and its management on the farm

The aim of this article is to discuss the consequences of disease on animal well-being and to consider the best ways of managing it on the farm. The physical well-being of the sick animal is jeopardised: the animal manifests stress-related physiological changes, adopts abnormal postures and movements to avoid pain, and has difficulty in carrying out normal behaviour patterns, and consequently its performance declines. Its mental well-being is also affected. Farmers and farm advisors need to become aware of the sick animal's suffering. Farmers need to detect and treat sick animals rapidly. The therapeutic procedures of veterinary surgeons need to be improved, with the use of analgesics as soon as the need arises.
\end{abstract}

BAREILLE N., 2007. Le mal-être de l'animal malade et sa gestion en élevage. INRA Prod. Anim., 20, 87-92. 